SUSX-TH-96-006

\title{
Bubble collisions in Abelian gauge theories and the geodesic rule.
}

\author{
E.J. Copeland* \& P.M. Saffin ${ }^{\dagger}$ \\ School of Mathematical and Physical Sciences, University of Sussex, \\ Brighton BN1 9QH, United Kingdom
}

(October 9, 2018)

\begin{abstract}
In an Abelian gauge symmetry, spontaneously broken at a first order phase transition, we investigate the evolution of two and three bubbles of the broken symmetry phase. The full field equations are evolved and we concentrate in particular on gauge invariant quantities, such as the magnetic field and the integral around a closed loop of the phase gradient. An intriguing feature emerges, namely, the geodesic rule, commonly used in numerical simulations to determine the density of defects formed is shown not to hold in a number of circumstances. It appears to be a function of the initial separation of the bubbles, and the coupling strength of the gauge field. The reason for the breakdown is that in the collision region the radial mode can be excited and often oscillates about its symmetry restoring value rather than settling to its broken symmetry value. This can lead to extra windings being induced in these regions, hence extra defects (anti-defects) being formed.
\end{abstract}

98.80. $\mathrm{Cq}$

${ }^{*} \mathrm{E}=$ mail: E.J.Copeland@sussex.ac.uk

†E-mail: p.m.saffin@sussex.ac.uk 
There is much evidence that the early Universe was characterised by a series of phase transitions in which a high energy 'old' symmetry phase was spontaneously broken to a low energy 'new' symmetry phase, possibly leading to the formation of topological defects. Such objects are also readily found in condensed matter systems (although of a much lower energy scale) [2], so although direct observational evidence for them in cosmology is still in doubt there exists plenty of evidence for their existence in terrestrial experiments. Given they could exist, we need to know their initial distribution so that we can determine the cosmological implications of the defects.

In this article we are concerned with the dynamics of Abelian-Higgs fields in a first order phase transition when bubbles of the new phase are nucleated in the background of the old phase. Topological defects form in the regions where the bubbles collide, so it would be useful to investigate the behaviour of the fields in this region. We will be following the work of [1] in determining the evolution of the bubble walls, but will be concerning ourselves with defect formation rather than the rate at which the energy in the bubble walls would be thermalized.

We will be considering a $U(1)$ theory with a complex scalar field $\Phi$, and Lagrangian

$$
\mathcal{L}=\left(D_{\mu} \Phi\right)^{*} D^{\mu} \Phi-\frac{1}{4} F_{\mu \nu} F^{\mu \nu}-V(\Phi)
$$

where $D_{\mu} \Phi=\partial_{\mu} \Phi-i e A_{\mu} \Phi, F_{\mu \nu}=\partial_{\mu} A_{\nu}-\partial_{\nu} A_{\mu}, e$ is the gauge coupling constant and the potential $V$ is a first order potential, a function of $|\Phi|^{2}$ with a local minimum at $|\Phi|=0$ and a global minimum at $|\Phi|=\eta / \sqrt{2}$. The dynamics of the phase transition proceed as follows. As the Universe expands and cools, tunnelling can occur from the old phase $\Phi=0$ to the new phase where $\Phi \simeq \frac{\eta}{\sqrt{2}} e^{i \theta}$. The symmetry has been spontaneously broken and within each bubble there is a random choice of the phase angle $\theta$. The bubbles, nucleated at random points expand and collide, with their nucleation rate being determined from the bounce solution to the Euclidean action [3].

The first definitive explanation for defect formation was provided in the work of Kibble [4]. Consider the case of cosmic strings. It is assumed that within each bubble the phase $\theta$ is constant, with neighbouring bubbles being uncorrelated. When two bubbles with phases $\theta_{1}$ and $\theta_{2}$ meet, any discontinuity between the phases is smoothed out. On energetic grounds, the shorter path between $\theta_{1}$ and $\theta_{2}$ on the vacuum manifold is chosen, a result known as the 'geodesic rule'. For the collision of three bubbles, a string may be trapped in the region between the bubbles. This depends on the net phase change in going sequentially $\theta_{1} \rightarrow \theta_{2} \rightarrow \theta_{3} \rightarrow \theta_{1}$ (see fig 8 ).

In a series of papers [5], Srivistava demonstrated that the geodesic rule does indeed hold for global theories. Recently though, with Rudaz [6], he questioned the reliability of the rule when gauge fields are present, pointing out that it may not make sense to talk about phase differences between bubbles in a gauge theory. It is possible to gauge transform the phase difference to any value. As a consequence they argued that string formation in such gauge theories may well be strongly suppressed as compared to the global theory case. Hindmarsh et al [7] analysed the bubble collisions using an analytic approach and concluded that the geodesic rule did actually hold. They pointed out that the geodesic rule emerges not from energy considerations but from the equations of motion themselves- the dynamics. Recently Kibble and Vilenkin [8], also using an analytic approach addressed the same issue, concluding that the geodesic rule nearly always held. They went further, including dissipation terms 
induced by the finite plasma conductivity that the bubbles expand in, they demonstrated that this can cause the phases to equilibrate on a timescale much smaller than the bubble radii at the time of collision. Such a result seems to vindicate the common assumption that the geodesic rule holds. However, as the authors stressed in [7] and [8], throughout the calculations various assumptions have to be made about the behaviour of the fields. For example in [8] it was assumed that the radial mode of the Higgs field is strongly damped, settling into its equilibrium value on a timescale short compared to the phase equilibrium process. A similar assumption was made in [7] where the variation of the radial mode was dropped inside the bubble. In general though, this may not happen.In this article we report on a project in which we solved the full field evolution equations for two colliding bubbles numerically, keeping track on the variation of the radial as well as the phase degrees of freedom. The results open up the possibility that the geodesic rule may not be as widespread as first thought.

Following [1] we consider a potential which is a function of $|\Phi|^{2}$ and which has a local minimum at the origin and a circle of degenerate global minima away from the origin. The simplest such potential is a cubic in $|\Phi|^{2}$, hence we consider

$$
V(\phi)=a\left(|\phi|^{2}+B\right)\left(|\phi|^{2}-c^{2}\right)^{2} .
$$

Two of the three parameters $(a, c)$ in Eqn. (2) can be set to unity by redefining the fields and coordinates.

The nucleation of a single bubble follows the bounce solution [3]. The gauge fields are taken to vanish and the phase is required to be constant within the bubble. The bounce solution found numerically was seen to compare favourably with a $\tanh (x)$ profile, which was subsequently used in the simulations as the initial condition on the Higgs field. (Our results are robust to this approximation due to the Lorentz contraction of the bubble walls.) In the problem of two bubbles colliding the tunnelling solution has $S O(2,1)$ symmetry and a suitable coordinate system to utilise this is

$$
\begin{aligned}
& t=s \cosh (\psi) \\
& y=s \sinh (\psi) \sin (\varphi) \\
& z=s \sinh (\psi) \cos (\varphi) \\
& x=x
\end{aligned}
$$

The fields must then evolve independantly of the trigonometric and hyperbolic angles $\varphi$ and $\psi$. This greatly simplifies the calculation since the solution becomes a function of two variables, the $\mathrm{x}$ coordinate, and the 'time' $s$ where $s^{2}=t^{2}-y^{2}-z^{2}$. A stationary point, $s, x=$ const, in these coordinates thus corresponds to an expanding loop in Minkowski space, with the $x$ axis as the symmetry axis.

There are two useful representations for $\Phi$. Firstly one has $\Phi=\frac{\rho}{\sqrt{2}} e^{i \theta}$. This is easier to visualise but difficult to implement numerically, due to the ambiguity in $\theta$ when the field modulus $\rho$ vanishes. Secondly there is a Cartesian description $\Phi=\frac{1}{\sqrt{2}}(u+i v)$, where $u, v$ are real. The choice of the Lorentz gauge $\left(\nabla_{\alpha} A^{\alpha}=0\right)$ and the independance of $\psi, \varphi$ leads to the set of evolution equations.

$$
\ddot{\rho}=\rho^{\prime \prime}-\frac{2}{s} \dot{\rho}+\rho\left[\dot{\theta}^{2}-\theta^{\prime 2}\right]-\frac{\partial \mathcal{V}}{\partial \rho}+e^{2} \rho\left[A_{s}^{2}-A_{x}^{2}\right]+2 e \rho\left[A_{x} \theta^{\prime}-A_{s} \dot{\theta}\right]
$$




$$
\begin{aligned}
\ddot{\theta} & =\theta^{\prime \prime}-\frac{2}{s} \dot{\theta}-\frac{2}{\rho}\left[\dot{\rho} \dot{\theta}-\rho^{\prime} \theta^{\prime}\right]+\frac{2 e}{\rho}\left[A_{s} \dot{\rho}-A_{x} \rho^{\prime}\right] \\
\ddot{A_{s}} & =A_{s}^{\prime \prime}-\frac{2}{s} \dot{A}_{s}+\frac{2}{s^{2}} A_{s}-e^{2} \rho^{2} A_{s}+e \rho^{2} \dot{\theta} \\
\ddot{A_{x}} & =A_{x}^{\prime \prime}-\frac{2}{s} \dot{A}_{x}-e^{2} \rho^{2} A_{x}+e \rho^{2} \theta^{\prime}
\end{aligned}
$$

Where $\dot{f}=\partial f / \partial s, f^{\prime}=\partial f / \partial x$. Note that there is a term in (8) depending on $e$, the gauge fields and the derivatives of $\rho$, which may be interpreted as a forcing term for $\theta$ and is the central difference between taking $\rho$ as constant [8] and considering the global case [5].

For the initial conditions of two bubbles with a phase difference, the description of the field's modulus is easy to understand. As the bubbles collide the intersection forms a ring, described by $s, x=$ constant, and the large amount of energy available in the walls at collision allows the $U(1)$ symmetry to be restored in this ring. It is this localised symmetry restoration which allows (but does not require) the phase to have a non-trivial winding about the ring, thus indicating the break down of the geodesic rule. Such a winding occurs in many cases. It is found that the amount of winding and its direction depends on the size of the gauge coupling, the initial separation and initial phase difference of the bubbles. By holding the phase and bubble separation constant we may investigate the $e$ dependance. When $e=0$, the global case, the winding around the ring is seen to vanish, consistent with the geodesic rule. Increasing $e$ leads to a generation of winding, which can flip sign from being positive to negative and also produce multiple $2 \pi$ windings. Subsequent flips of sign occur for values of $e$ which are integer multiples of the value of $e$ for which the flip first occurs.

The dependance on initial separation is probed by holding $e$ and the phase difference constant. It is found that the greater the separation, the greater the winding. As for the dependance on the initial phase separation, we observe that there is a cutoff around $\frac{\pi}{2}$ above which no winding is generated.

The picture describing the evolution of the Higgs field is shown for example in Figure 1. Two regions of true vacuum expand, collide and form a pocket of restored $U(1)$ symmetry which subsequently collapses and forms another pocket. These oscillations continue until the energy has been radiated away [1].

A non-trivial winding is observed to occur over the time period that the first pocket collapses (see Figure 2). This winding occurs at constant $s, x$ inside the true vacuum region and so corresponds to an expanding loop following the intersection of the two bubbles. This loop has a unit winding and so corresponds to an expanding loop of string. This timescale can be estimated [1] by considering the thin wall approximation for a global theory. Let $s_{0}$ be the coordinate time at which the walls collide and $s_{2}$ when the pocket collapses. By matching the wall velocities before and after collision (consistent with simulations) then it is possible to show:

$$
s_{2} \simeq\left[2\left(2\left[1-\frac{1}{3} \alpha^{2}\right]\right)^{\frac{1}{3}}-1\right] s_{0}
$$

where $\alpha$ is the phase separation between the bubbles. The limiting case is when $s_{2} \simeq s_{0}$ for which we find a value of $\alpha$ of $\simeq \sqrt{\frac{3}{2}} \simeq \frac{\pi}{2}$, a result that explains why no windings are 
found above a certain initial phase separation. This phase angle cutoff is due to the extra energy in the phase wave that propogates away from the collision region, leaving insufficient energy to restore the symmetry. Shortly we shall see that the amount of winding depends on how long this first pocket can survive. From Eqn. (11) the lifetime varies in proportion to $s_{0}$ so, since the bubbles reach relativistic velocities rapidly, the pocket's lifetime will vary in proportion to the initial separation, a result that explains why more windings were found as the separation was increased.

Now we consider the cause of the winding. If we set the phase in the left hand bubble to be zero and in the right hand bubble to be $0<\alpha<\pi$, the equations of motion require $A_{x}>0$ for all $x$ and $A_{s}<0$ for $(x>0)$ just after the collision. As the pocket initially expands then $\dot{\rho}<0, \rho^{\prime}>0$ which means the term $\frac{2 e}{\rho}\left(A_{s} \dot{\rho}-A_{x} \rho^{\prime}\right)$ in Eqn. (8) has components which oppose each other and lead to a small forcing term for $\theta$. However, when the pocket starts to collapse then $\dot{\rho}>0$ and the components combine to create a negative forcing term for $\theta$, driving the field around the true vacuum manifold and generating a winding (see Figures $2,5)$. Note that the longer the pocket survives, then the longer this forcing can act leading to more winding. An important feature of the winding is that as the bubbles collide and the radial component of the field overshoots the global minima restoring the symmetry, the field traces a twist in the configuration space which leads to a winding of $+2 \pi$ instead of the $-2 \pi$ which would occur without the twist (see Figures 4,5 at $\mathrm{s}=31.5, \mathrm{~s}=32$ ).

The sense of the winding depends upon the sign of the gauge fields. If it is possible to change the sign of the gauge fields by the time the pocket starts to collapse then the winding direction is reversed. In equations (9, 10) we find the harmonic term $-e^{2} \rho^{2} A$ which can generate a sign change on a timescale of $\frac{\pi}{e \rho}$. This scale is only significant for large $\rho$ and should be compared with the timescale of the modulus wall, $\delta$. If the gauge fields $A_{s}, A_{x}$ can change sign in the time taken for the modulus to drop to zero, then they will retain their new sign until the pocket collapses. This leads to a change in the sense of the winding at $e \simeq \frac{\pi}{\eta \delta} n$, where $n$ is an integer. This demonstrates the sign flipping observed in the simulations at regular intervals of $e$ and agrees numerically with the observed periodicity.

With a constant field modulus in the true vacuum, $\rho=\eta$, then the field equations have the solution $e A_{\mu}=\partial_{\mu} \theta$. Using the two bubble collision depicted in fig6, we may evaluate the associated magnetic flux of the winding. As the gauge field vanishes on sides $\mathrm{BC}, \mathrm{CD}, \mathrm{DA}$ then the magnetic flux, $\Phi=\oint \underline{A} \cdot \underline{d l}$, inside the loop ABCD is $\Phi_{A B C D}=\int_{A}^{B} A_{x} \mathrm{~d} x$. When the collision region (the intersection of the two bubbles) has moved from the $\mathrm{x}$ axis then along the $x$ axis $e A_{\mu} \simeq \partial_{\mu} \theta$ and so $\Phi \simeq \alpha_{12} / e+2 \pi n_{12} / e$. The value of the integer $n_{12}$ represents the extra winding and depends on how the geodesic rule is broken, with the geodesic rule requiring $n_{12}$ to vanish. From the simulations it was found that couplings of $\mathrm{e}=0.1,0.5$, $1.0,1.5$ (with an initial separation of 60 units and phase difference of $\alpha_{12}=\pi / 4$ ) lead to extra windings of $0,1,2,-1$ respectively. The flux measured through the loop ABCD for these couplings is shown in fig7, in units of $2 \pi / e$. The magnetic flux, which is a gauge invariant quantity, through the enclosed region is seen to oscilate about $2 \pi / e\left(1 / 8+n_{12}\right)$ with $n_{12}=0,1,2,-1$ thus confirming the expected flux. The late time oscillations correspond to the natural frequency $\omega=e \eta$.

It is important to know whether this breaking of the geodesic rule rule can lead to stable defects. As explained, the collision region forms a ring and this ring can have a winding, i.e. a loop of string. This loop is close to the false vacuum surrounding the bubbles but as 
seen in fig2 it cannot escape into the false vacuum, wiping out the string. In order to form a string surrounded by true vacuum we need at least three bubbles, we now consider how the above can alter the predictions for these trapped strings. For a path $\Gamma$ (fig8) which is outside the collision regions then the field modulus assumes a non zero value so that the phase is defined on each segment of the path. The path $\Gamma$ also has the property that any leg of the path joining two bubbles has not been reached by the third bubble. This means that we may treat the phase on each leg of $\Gamma$ simply by considering the relevant two bubble collision. The total winding enclosed in this path is then

$$
n_{t}=\frac{1}{2 \pi}\left(\alpha_{12}+\alpha_{23}+\alpha_{31}\right)+n_{12}+n_{23}+n_{31}
$$

where $n_{i j}$ is the extra winding induced by the gauge field between bubbles $i$ and $j$. As an example, consider bubbles with $\theta_{1}=0, \theta_{2}=\pi / 4$ and $\theta_{3}=\pi / 2$, all initially separated by 60 units with a gauge coupling of $e=0.5$. From figs 2,5 we know that as $\alpha_{12}=\alpha_{23}=\pi / 4$, then $n_{12}=n_{23}=1$. Also, because $\alpha_{31}=\pi / 2$ then no extra winding is expected between bubbles 1, 3 due to the upper bound on $\alpha$ for extra winding caused by the energy in the phase wave, so $n_{31}=0$. The total winding thus expected is two, whereas the geodesic rule would have predicted no winding. The result of a simulation is shown in fig9. The phase is seen to change by $4 \pi$ as we traverse the sides of the triangle and this leads to the presence of the two single winding vortices which can be seen in the figure. The existence of a path, $\Gamma$, where the field is away from the collision area is important. If such a path cannot be drawn then it may be possible for the extra windings to escape.

The effects of dissipation are expected to play a significant rôle in this mechanism, as the field is required to have enough energy locally to temporarily restore the symmetry. If this energy can be dissipated away on short enough timescales then the geodesic rule will be restored.

Following ref [8], we modelled the effects of dissipation using an Ohmic current, defined through the projection relation

$$
\left[g_{\nu}^{\mu}-U_{\nu} U^{\mu}\right] j_{O h m}^{\nu}=-\sigma U_{\nu} F^{\nu \mu}
$$

where $U$ is the fluid four velocity. We then use the conservation constraint $\nabla_{\mu} j_{O h m}^{\mu}=0$ to fully define the current and rewrite the gauge field equations of motion as $\partial_{\mu} F^{\mu \nu}=j_{\text {Noether }}+j_{O h m}$. The effects of so doing are seen in fig3 where, for the parameters, used in figs 2,5 the winding has reverted back to the geodesic rule when the conductivity, $\sigma=0.5$. As the conductivity of the plasma is increased then the more the gauge field gets coupled to the Ohmic current rather than the Noether current, the effect of this is to reduce the gauge field forcing term of equation (8) and, for a large enough conductivity, the geodesic rule is restored.

On the face of it the cosmological implications of a breakdown of the geodesic rule could be very significant. Typically, as mentioned earlier, the phases are set at random in each bubble and the geodesic rule is used to determine the density of defects formed initially. This leads to a distribution of open and closed string. Now we seem to find that depending on the parameters, if the phase difference between two bubbles is less than about $\pi / 2$, then it is likely that new windings will be induced in the collision region. A technique has recently been developed [10] in which the length distribution of strings can be calculated numerically, 
without a regular lattice dependance. This method is particularly suited to modelling a first order phase transition and it would be interesting to extend those results to take into account the effects described above.

There is a precedent for extra vortices being found in the collision regions of the bubble walls. Digal and Srivastava [11] have analysed the behaviour of two bubbles colliding in a global $\mathrm{U}(1)$ theory (in $2+1$ dimensions) where the global symmetry is broken both spontaneously and explicitely. They find that in the coalesced region of the bubbles, field oscillations result in the production of a number of defects (vortices and anti-vortices). We have been able to confirm this behaviour in the case of $3+1$ dimensions and will report on this elsewhere [12].

In conclusion, the results we have obtained suggest that a set of rules exist for predicting the likely outcome of defects after two bubble collisions. By calculating gauge invariant quantities, like the magnetic flux and closed loop integral of the phase gradient, we have shown how the number of windings found varies. The phase winding of the loop was seen to depend on the initial separation of the bubbles, the strength of the coupling constant $e$, and on the currently unknown strength of the dissipation term, $\sigma$. A point to stress is that the rule determining winding will be one that depends on the dynamics of the fields, not one determined from energetic considerations, a comment also made in [7]. The loops that are generated in this process are extra vortices, the final state of the phase transition is different to that predicted with the geodesic rule. An important and as yet unresolved issue concerns the statistical length distribution of string loops in the network of defects. Naively there would appear to be far more loops produced which do not simply shrink away. As has been pointed out by Borrill [10] such a result could have implications for the normalisation of the string tension. We have shown that these extra loops of string are expected when the phase separation is less than $\simeq \pi / 2$. This will be the case on average for around half of the bubble pairings and may therefore significantly change the length distribution of string.

\section{ACKNOWLEDGMENTS}

We are grateful to J. Borrill, M. Hindmarsh, R. Holman, T.W.B. Kibble and A.M. Sri-

vastava for useful comments and conversations. P.M.S. is grateful to G.Vincent for tutoring in $\mathrm{C}$ and to PPARC for financial support. 


\section{REFERENCES}

[1] S.W. Hawking, I.G. Moss \& J.M. Stewart, Pays. Rev. D26, 2681 (1982).

[2] For a review see 'Formation and Interactions of Topological Defects', (NATO ASI Series B: Physics Vol 349, Eds: A.C. Davis \& R. Brandenberger (1995)).

[3] S. Coleman, Phys. Rev. D15, 2929, (1977).

[4] T.W.B. Kibble, J. Phys A: Math. \& Gen. 9, 1387 (1976).

[5] A.M.Srivastava, Phys.Rev.D45, R3304 (1992); Phys.Rev.D46, 1353 (1992).

[6] S. Rudaz \& A.M. Srivastava, Mod. Phys. Lett. A8, 1443 (1993)

[7] M. Hindmarsh, A.C. Davis \& R. Brandenberger, Phys. Rev. D49, 1944 (1994)

[8] T.W.B. Kibble \& A. Vilenkin, Phys. Rev. D52, 679 (1995).

[9] T. Vachaspati \& A. Vilenkin, Phys. Rev. D30, 2036 (1984); D. Austin, E.J. Copeland \& R.J. Rivers, Phys. Rev. D49, 4089 (1994).

[10] J. Borrill, Phys. Rev. Lett. 76, 3255 (1996)

[11] S. Digal \& A.M. Srivastava, Phys. Rev. Lett. 76, 583 (1996)

[12] E.J.Copeland \& P.M.Saffin in preparation.

\section{List of Figures}

Figure 1. Field evolution plot for $e=0.0,0<s<60,-30<x<30$. The bubbles are nucleated at $(s, x)=(0, \pm 30)$ The magnitude of the Higgs field is represented by the arrow length and the phase by its direction.

Figure 2. Field evolution plot for $e=0.5,0<s<60,-30<x<30$ where a winding of $2 \pi$ is generated.

Figure 3. Field evolution plot for $e=0.5, \sigma=0.50<s<60,-30<x<30$ where a winding of $2 \pi$ is prevented due to the dissipation of the energy in the gauge field.

Figure 4. Sequence in field configuration $(u, v)$ space for $e=0.0$. and $\phi=1 / \sqrt{2}(u+i v)$ along the path $-30<x<30$.

Figure 5. Sequence in field configuration $(u, v)$ space for $e=0.5$. and $\phi=1 / \sqrt{2}(u+i v)$ along the path $-30<x<30$.

Figure 6. Schematic representation of a configuration for two colliding bubbles with phases $\theta_{1}, \theta_{2}$ and phase difference $\alpha_{12}$ showing the path used to measure the magnetic flux.

Figure 7. Magnetic flux through the region depicted in fig6 for various gauge couplings as a function of $s$. The initial conditions are two bubbles nucleated at a separation of 60 units with a phase separation of $\pi / 4$.

Figure 8. Schematic representation of a three bubble collision showing bubbles with phases $\theta_{i}$ and phase separations $\alpha_{i j}$.

Figure 9. Plot of a three bubble collision in the $x-y$ plane with $e=0.5$ showing the generation of two stable windings which contradicts the prediction of the geodesic rule. The three bubbles (with initial phases $0, \pi / 4, \pi / 2$ ) were nucleated at equal times with their centres at the vertices of the equalateral triangle. The figure represents the state which such a configuration will evolve to. 


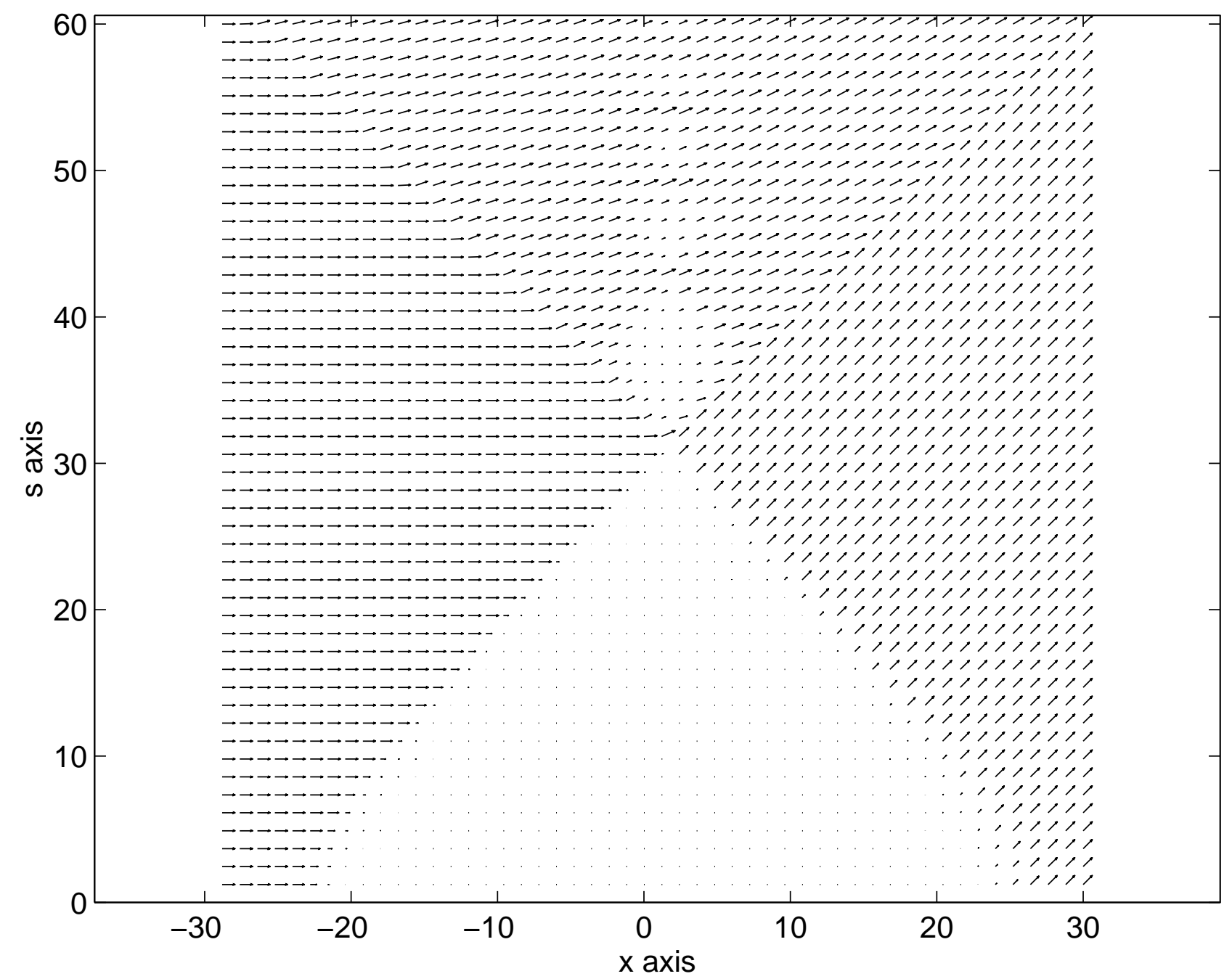




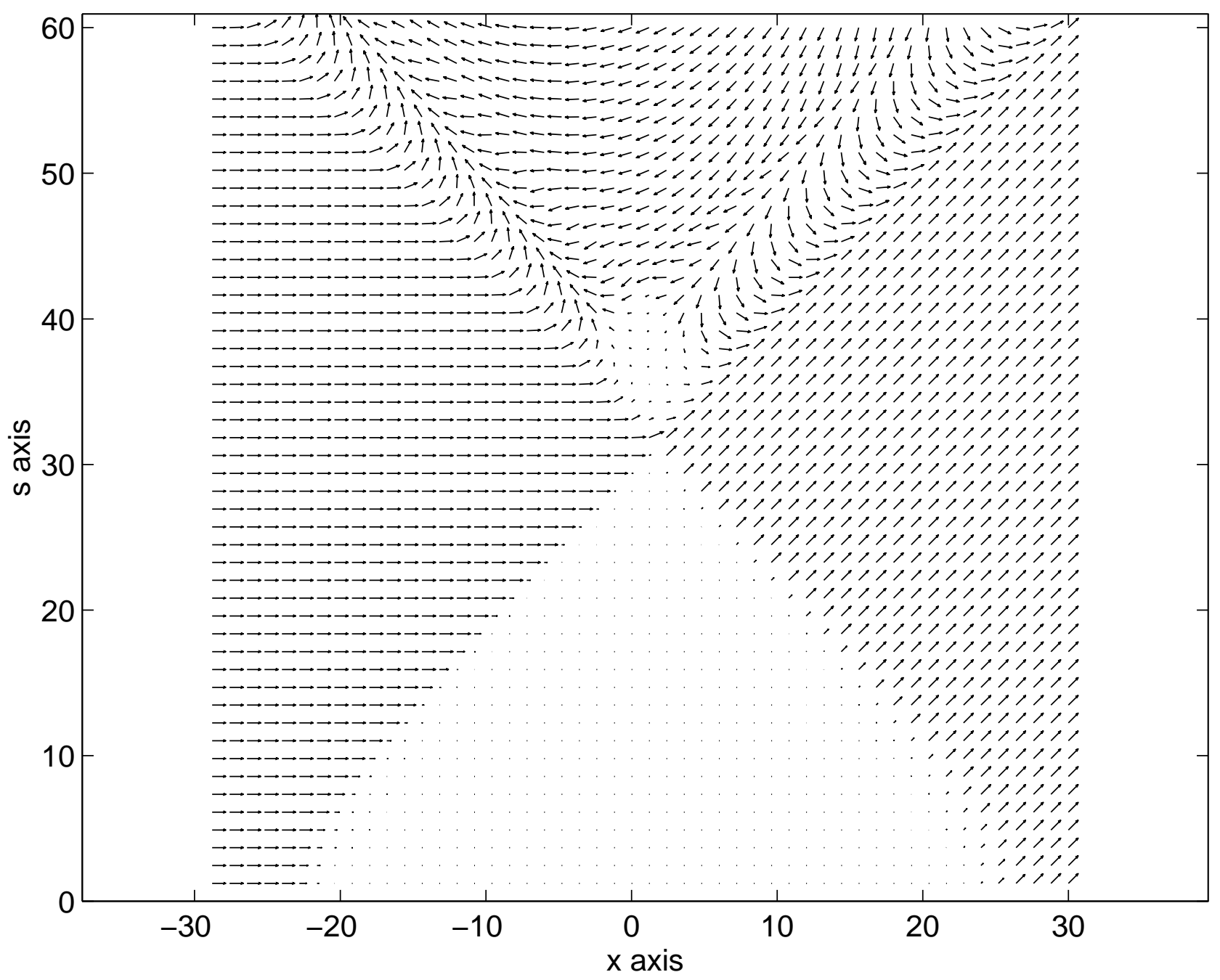




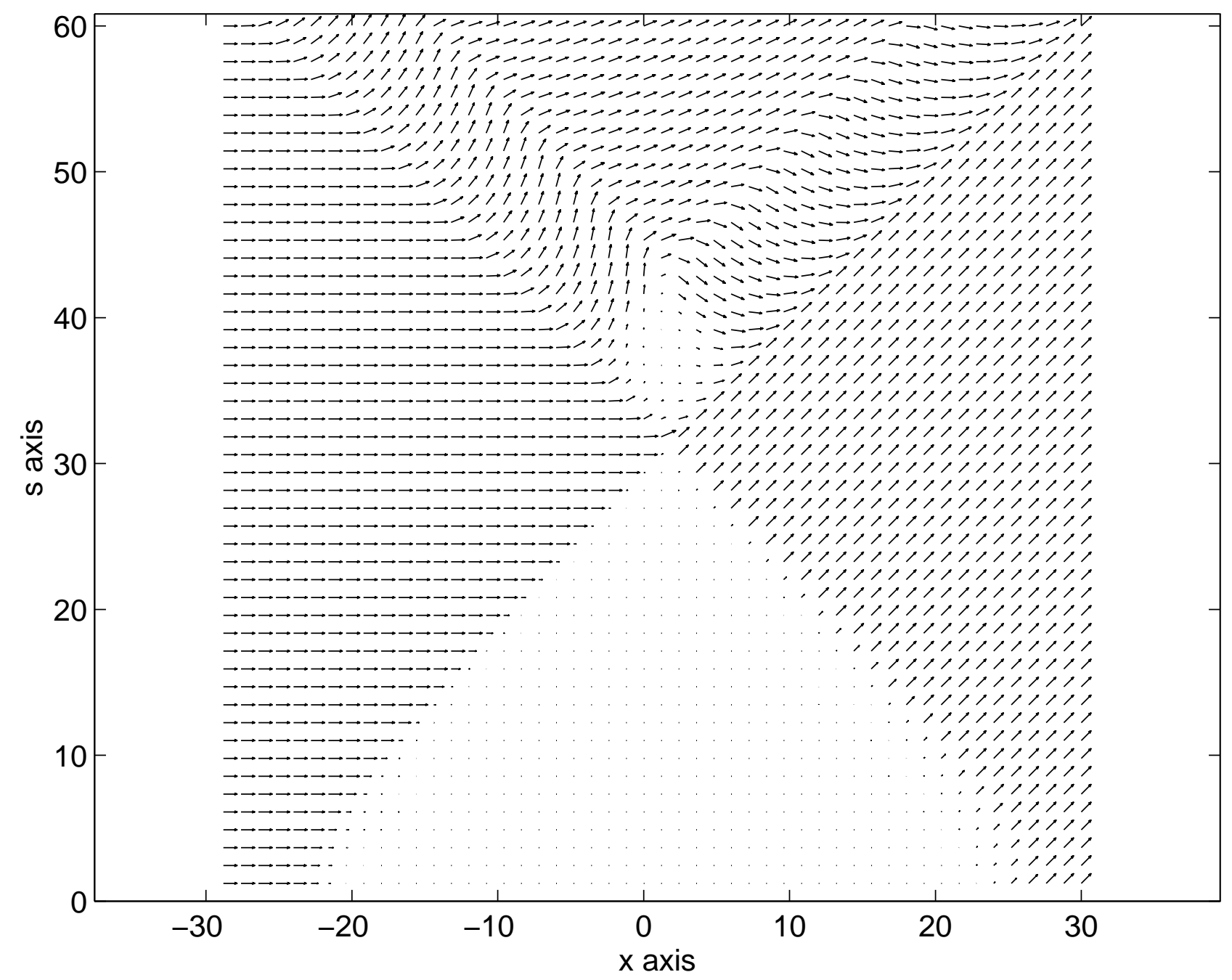



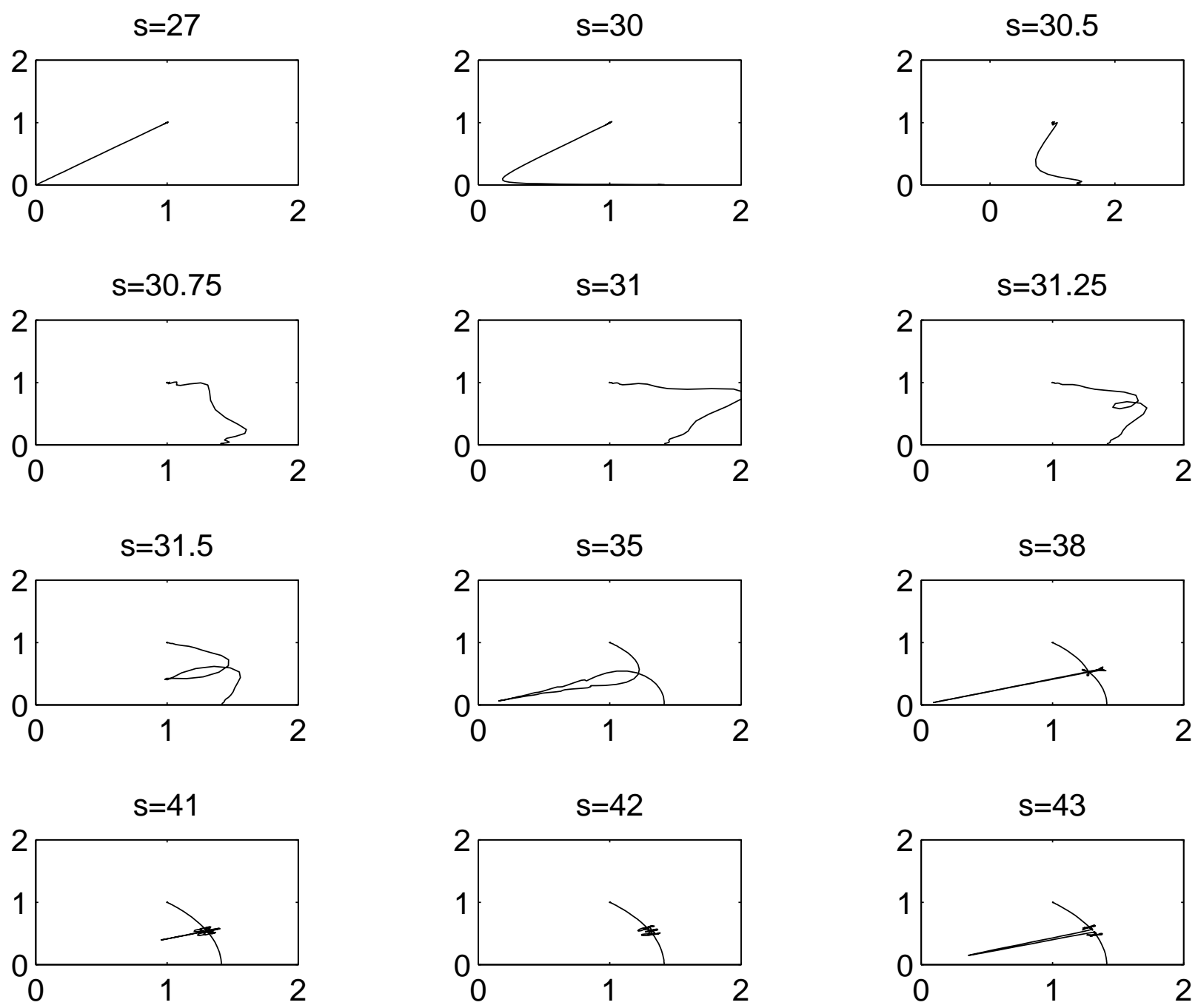

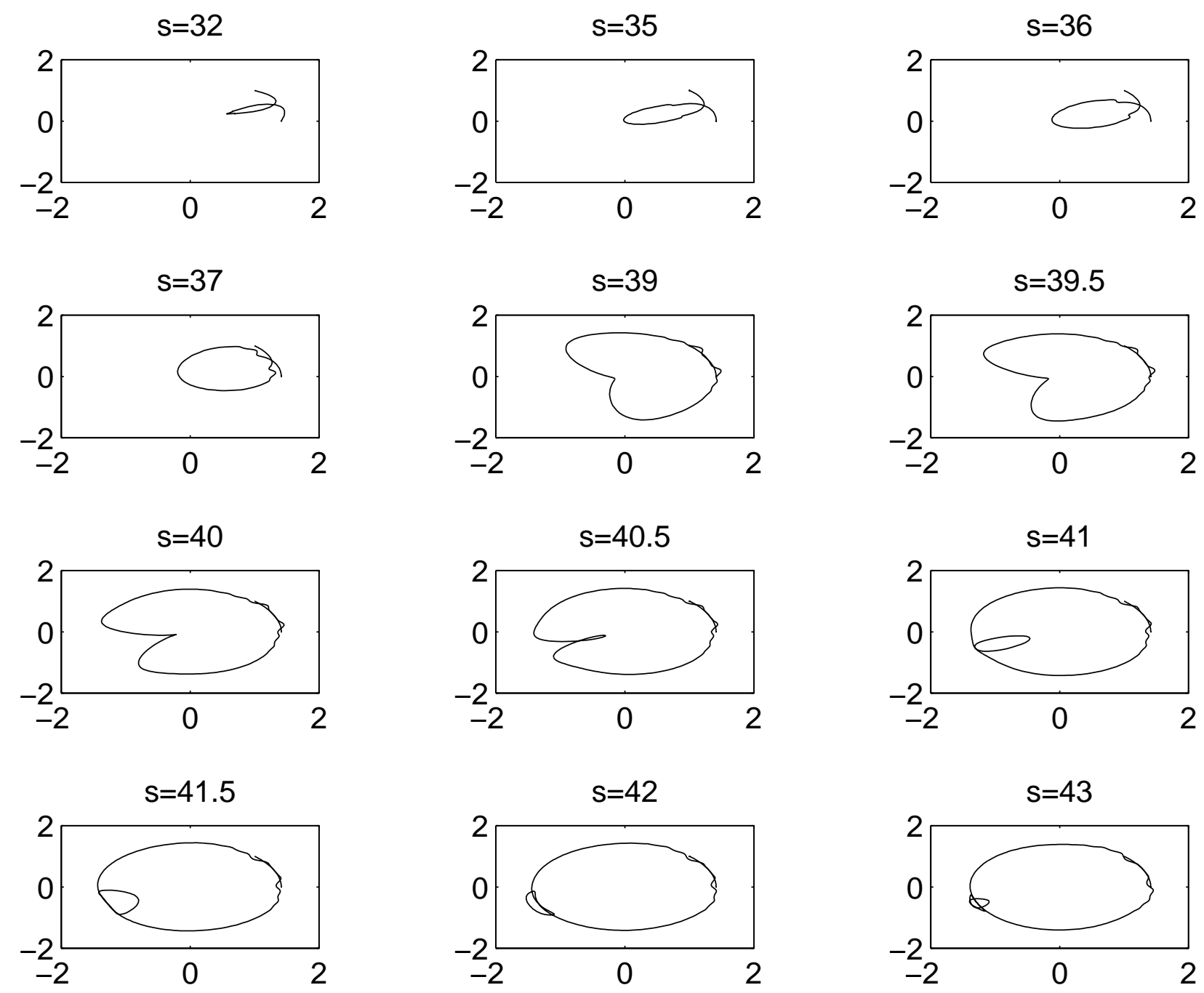

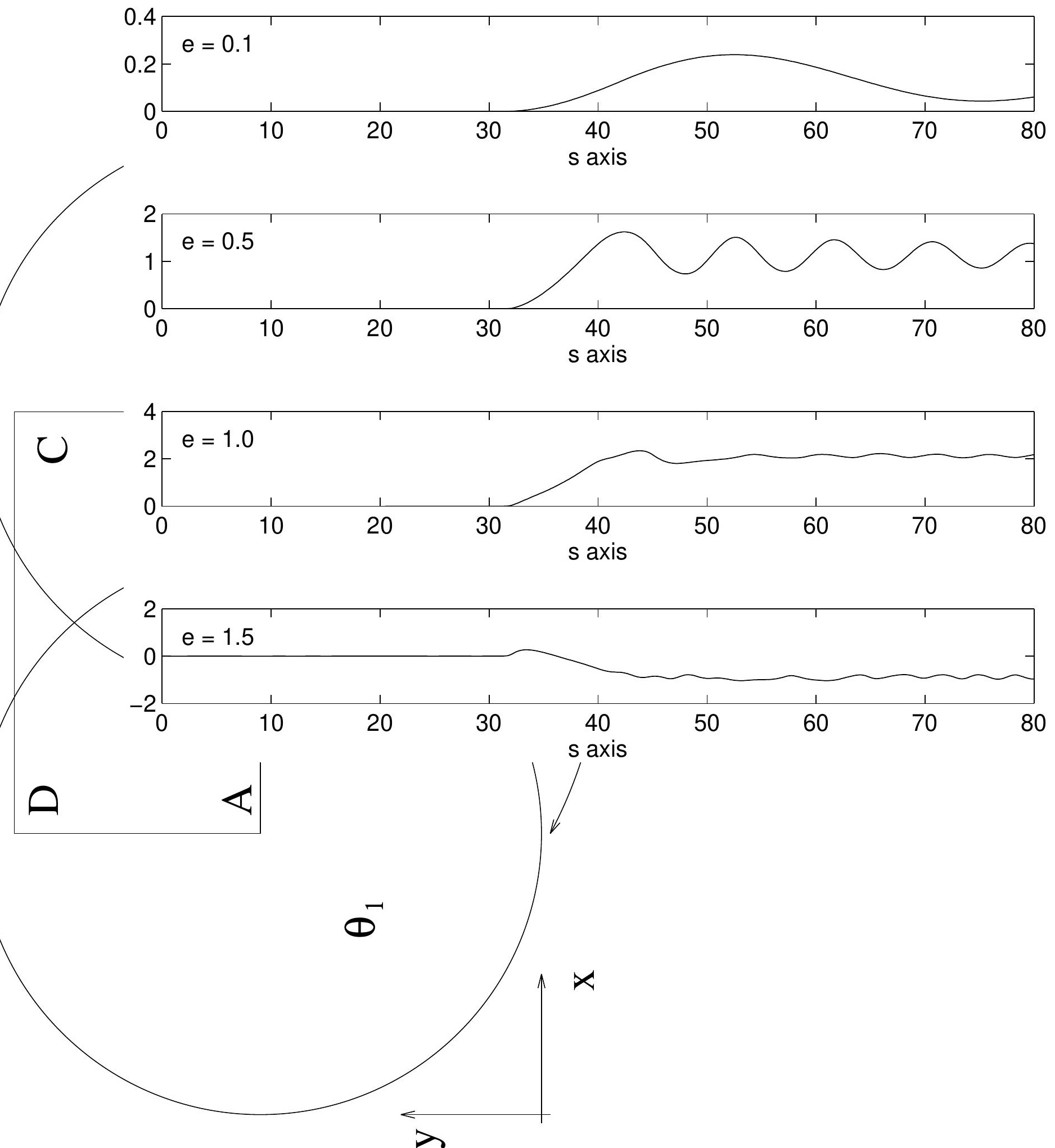

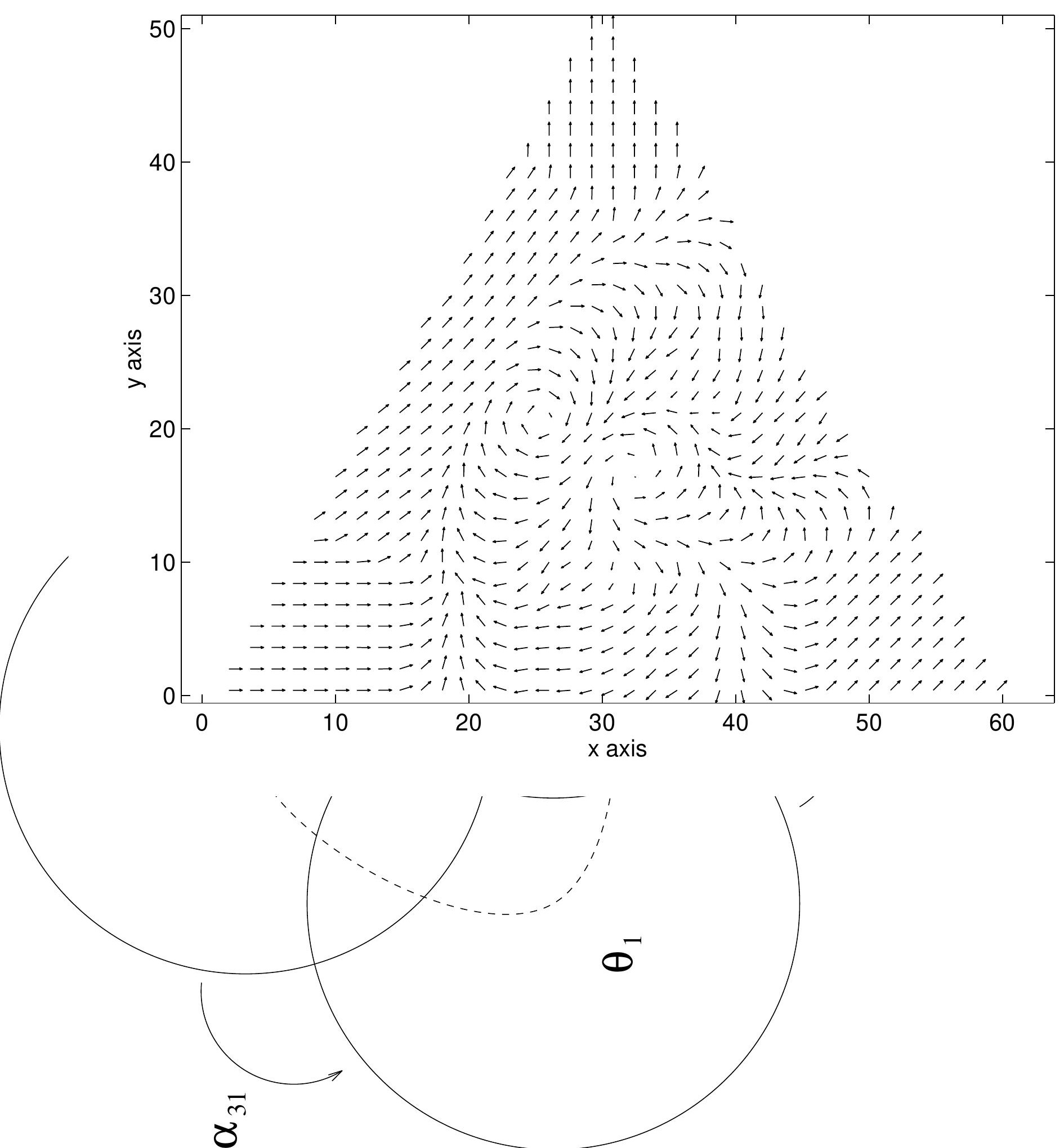